\title{
Die bayerischen Gletscher, die verbliebenen Eisreserven Deutschlands
}

\author{
Christoph Mayer ${ }^{1}$, Markus Weber ${ }^{2}$, Anja Wendt ${ }^{1}$, and Wilfried $\mathrm{Hagg}^{3}$ \\ ${ }^{1}$ Erdmessung und Glaziologie, Bayerische Akademie der Wissenschaften, München, Deutschland \\ ${ }^{2}$ Lehrstuhl für Photogrammetrie und Fernerkundung, Technische Universität München, München, Deutschland \\ ${ }^{3}$ Fakultät für Geoinformation, Hochschule München, München, Deutschland \\ Correspondence: Christoph Mayer (christoph.mayer@badw.de)
}

Published: 20 May 2021

Kurzfassung. Gletscher gibt es in Deutschland nur in dem sehr kleinen Alpenanteil und dort nur an wenigen, für die Eismassen günstigen Stellen. Diese Gletscher sind vermutlich keine Relikte der letzten Eiszeit, sondern Reste der kühleren Phasen der letzten Jahrhunderte. Trotzdem ging von diesen verbliebenen Eisreserven seit dem Beginn der touristischen Erschließung der Alpen eine große Faszination aus, da sie teilweise vom Tal aus zu sehen, oder verhältnismäßig leicht zu erreichen waren. Selbst heute noch kann man auf dem nördlichen Schneeferner den einzigen Gletscherskilift in Deutschland erleben, wenngleich auch dort die Gletscherflächen erheblich zurückgegangen sind. Die frühe Aufmerksamkeit führte auch dazu, dass es viele historische Dokumente über diese Gletscher gibt und so zumindest ihre Veränderung seit dem Ende der kleinen Eiszeit gut dokumentiert ist. Heute sind fast alle der bayerischen Gletscher am Rande ihrer Existenz und es ist nur eine Frage der Zeit, bis der deutsche Alpenraum eisfrei wird. Wir versuchen mit diesem Beitrag die Entwicklung der Gletscher seit den ersten Untersuchungen zu dokumentieren.

\section{Die bayerischen Gletscher}

Die bayerischen Gletscher hatten 2018 eine Gesamtfläche von 44,6 ha und ein Volumen von 3,95 Mio. $\mathrm{m}^{3}$. Seit der letzten Erhebung im Jahr 2009 haben die Gletscher $62 \%$ ihres Volumens verloren (Mayer und Hagg, 2018). Dieser Verlust in nur neun Jahren deutet schon an, dass die klimatischen Bedingungen für die Existenz von Gletschern im deutschen Alpenraum nicht mehr gegeben sind. Die klimatische Schneegrenze liegt schon seit Jahrzehnten oberhalb der Gipfelhöhen
(Hagg, 2008) und die zunehmende Veränderung des Klimas setzt den Gletschern stark zu.

Derzeit gibt es in den bayerischen Alpen noch fünf Gletscher an der Zugspitze und in den Berchtesgadener Alpen. Dabei wird hier nicht explizit auf die Gletscherdefinition als mehrjähriges Eisvorkommen mit Anzeichen von Eisdeformation zurückgegriffen, da einige dieser Eiskörper sich schon in der Transformation zu Gletscherresten befinden. Die fünf Gletscher sind:

1. Der Nördliche Schneeferner (NSF) ist der zweitgrößte, aber immer noch höchstgelegene Gletscher Bayerns. Aufgrund der Lage direkt östlich des Grates zwischen Zugspitze und Schneefernerkopf treten dort sehr hohe Niederschläge auf, die auch die ursprüngliche Ursache für die Bildung des Gletschers waren. Seit 1979 schmilzt der nördliche Schneeferner wie alle bayerischen Gletscher kontinuierlich zurück, was sich jedoch durch die Muldenlage lange Zeit nicht in deutlichen Flächenänderungen manifestiert hat. Inzwischen hat er allerdings einen Großteil seiner Zunge verloren, da die Eisdicken im unteren Bereich deutlich kleiner waren als im Zentrum. Auch der Skibetrieb beeinflusst den Gletscher. Deutlich ist die künstliche Zugangsrampe vom Zentrum Sonnalpin in die Mulde des Schneeferners zu erkennen (Abb. 1b).

2. Der Südliche Schneeferner (SSF, Abb. 1c) ist fast nicht mehr existent, obwohl er kurz nach dem Zerfall des Plattachferners der größte Gletscher in Bayern war. Im Gegensatz zum nördlichen Scheeferner, bei dem sich der Schnee in einer großen Mulde sammeln konnte und welche auch teilweise für eine zusätzliche Abschattung sorgt, besteht das Gelände des südlichen Schneeferners 


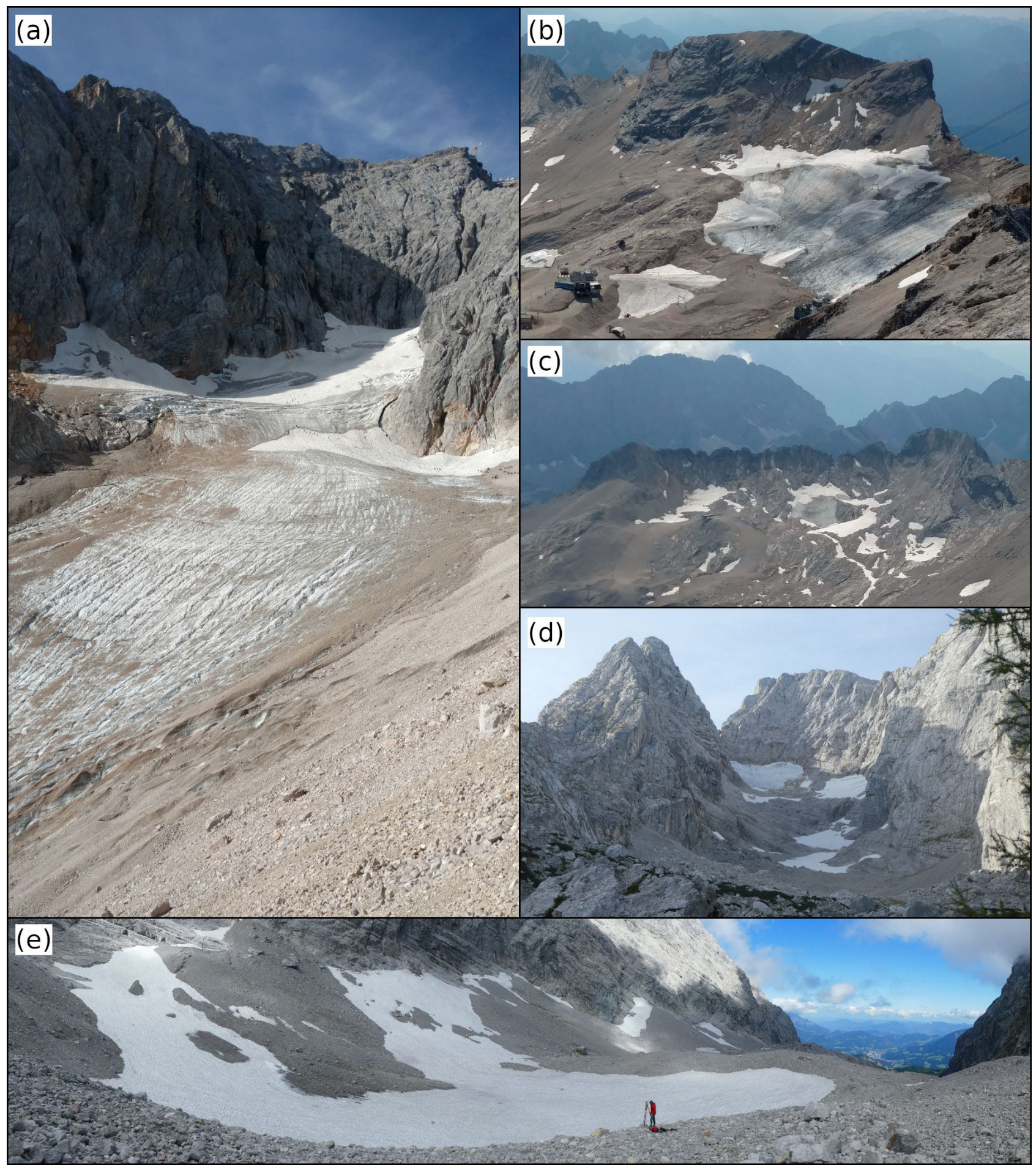

Abb. 1. Die bayerischen Gletscher: (a) Höllentalferner im September 2016; (b, c) nördlicher bzw. südlicher Schneeferner im September 2016 von der Bergstation der Zugspitzbahn aus; (d) die einzelnen Teile des Blaueises im September 2019 von der Blaueishütte; (e) Watzmanngletscher im September 2019 während einer Routinevermessung. Zu diesem Zeitpunkt war der Gletscher im Gegensatz zu den Vorjahren noch mit Schnee bedeckt. Fotos: (a)-(c) Anne Nowottnick, (d) Christoph Mayer, (e) Astrid Lambrecht. 
aus leicht hügeligem Gelände, wo sich größere Schneeansammlungen nur in kleinen Nischen sammeln konnten. Daher verfügte er über deutlich geringere Eisdicken und die Flächenverluste sind sehr ausgeprägt. Heute erhalten sich Teile der Eisreste nur noch unter den sommerlichen Schneedepots der Skigebietsbetreiber.

3. Der Höllentalferner (HTF) ist inzwischen der größte Gletscher Bayerns. Er liegt in einer tiefen Senke nördlich des Zugspitzgipfels und wird nach Süden hin von mehr als $300 \mathrm{~m}$ hohen Felswänden beschattet. Auch nach Westen und Nordwesten ist der Gletscher von hohen Felswänden eingerahmt. Die hohen Niederschläge auf der windabgewandten Seite und die Beschattung ermöglichen die Existenz des Gletschers, etwa $300 \mathrm{~m}$ tiefer und damit in einer wärmeren Höhenlage als der nördliche Schneeferner. Er verfügt als einziger der Gletscher noch über eine Gletscherzunge (das schmal auslaufende untere Ende eines Gletschers) und zumindest zeitweise über einen Akkumulationsbereich, wo im Jahresverlauf mehr Schnee liegen bleibt als geschmolzen wird. Ein wesentlicher Teil dieses Schnees stammt von Lawinenabgängen, ähnlich wie am Blaueis. Es ist auch der einzige Gletscher, der noch eine deutliche Gletscherbewegung zeigt, die im mittleren Teil des Gletschers in einigen großen Spalten resultiert. Die Zunge des Gletschers wird zunehmend mit ausschmelzendem Schutt bedeckt. Deutlich sind die tiefen Schmelzwasserrinnen auf der Gletscherzunge zu erkennen (Abb. 1a), welche auf hohe Schmelzraten hinweisen. Im Gletschervorfeld des Höllentalferners ist die Ausdehnung der neuzeitlichen Gletschervorstöße durch Moränenwälle gut dokumentiert.

4. Der Watzmanngletscher (WMG, Abb. 1e) befindet sich östlich der Mittelspitze des Watzmanns in den Berchtesgadener Alpen und ist mit einer mittleren Höhe von nur $2060 \mathrm{~m}$ über dem Meer der tiefstgelegene Gletscher in Bayern. Der Watzmanngletscher reagiert deutlicher als die anderen Gletscher auf klimatische Veränderungen. In der Wärmeperiode vor 1949 ist er in einzelne Firnflecken zerfallen, hat aber danach, als alle anderen Gletscher zwischen 1949 und 1959 noch erheblich an Masse verloren, bereits einen Zuwachs gezeigt. Der Grund dafür ist allerdings nicht klar. Inzwischen erstreckt sich das Eis jedoch nur noch in der Karmulde, sowie der Flanke direkt unter dem Watzmann. Diese Flanke ist stark schuttbedeckt und daher gut vor der Sonneneinstrahlung geschützt.

5. Das Blaueis (BEI, Abb. 1d) ist in einen tiefen Einschnitt nördlich des Gipfels des Hochkalter eingebettet und ist der nördlichste Gletscher der Alpen. Durch seine Lage ist er fast das gesamte Jahr weitgehend vor Sonneneinstrahlung geschützt. Die steile Felsumrahmung stellt ein großes Einzugsgebiet für Lawinenschnee dar.

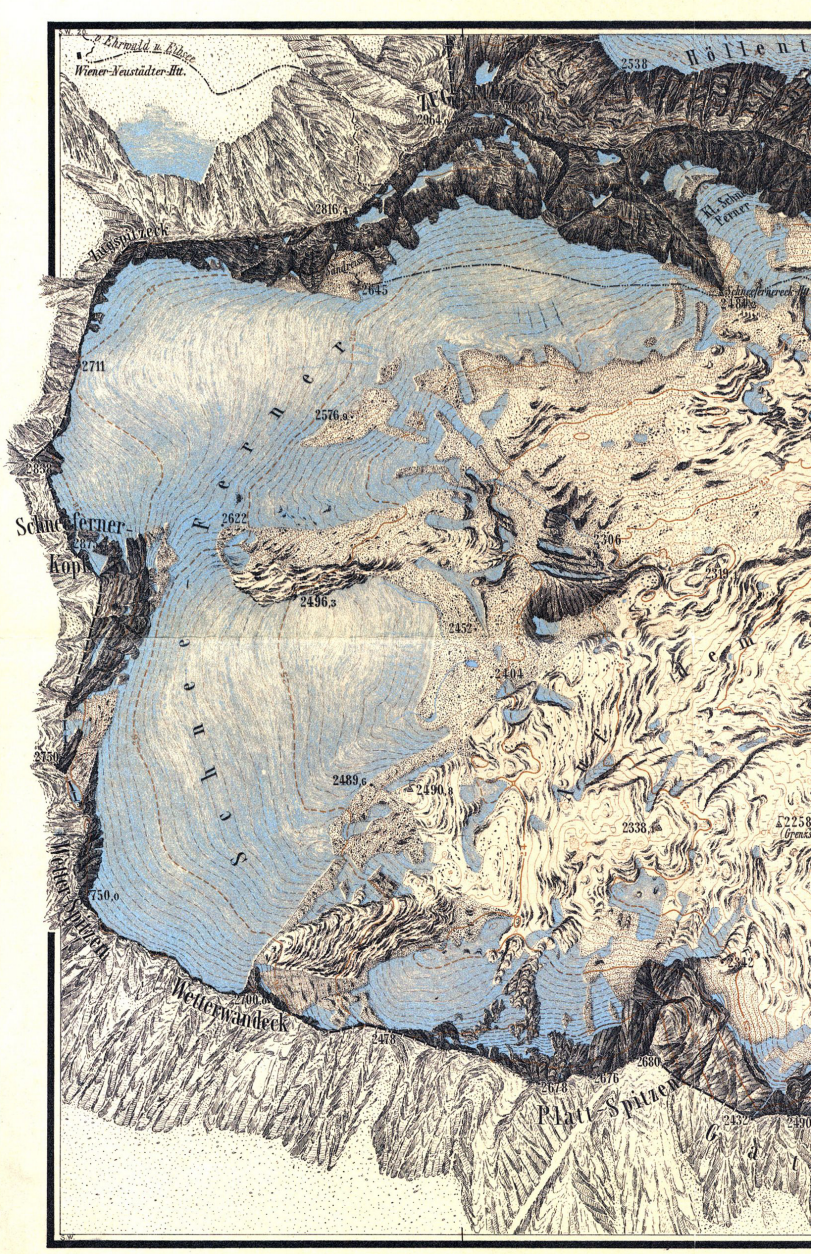

Abb. 2. Ausschnitt des Kartenblattes „Zugspitze“ von Finsterwalder und Jäger aus dem Jahr 1892 (Archiv, Erdmessung und Glaziologie, BAdW München).

In den letzten Jahren hat sich der Gletscher mehrfach durch ausschmelzende Felsriegel aufgeteilt. Die unterste Teilfläche ist schon seit Jahren mit Schutt bedeckt, erhält auch keinen Nachschub aus Akkumulation oder Eistransport und schmilzt daher langsam aber stetig ab. Auch die oberen Teile des Gletschers weisen nur in seltenen Jahren eine Schneerücklage im Herbst auf.

\section{Historische Beobachtungen}

Von den bayerischen Gletschern ist vermutlich der „Plattacher Ferner" als erstes kartographisch dokumentiert worden. Auf einem Kartenblatt des Atlas Tyroliensis von 1774 ist auf dem Zugspitzplatt ein Gletscher mit diesem Namen verzeichnet. Damals war die gesamte Hochfläche mit Gletschereis bedeckt, welches sich über eine Fläche von etwa 300 ha erstreckte (Finsterwalder, 1951). Von diesem damals größten Gletscher Bayerns sind heute nur noch die beiden Schnee- 

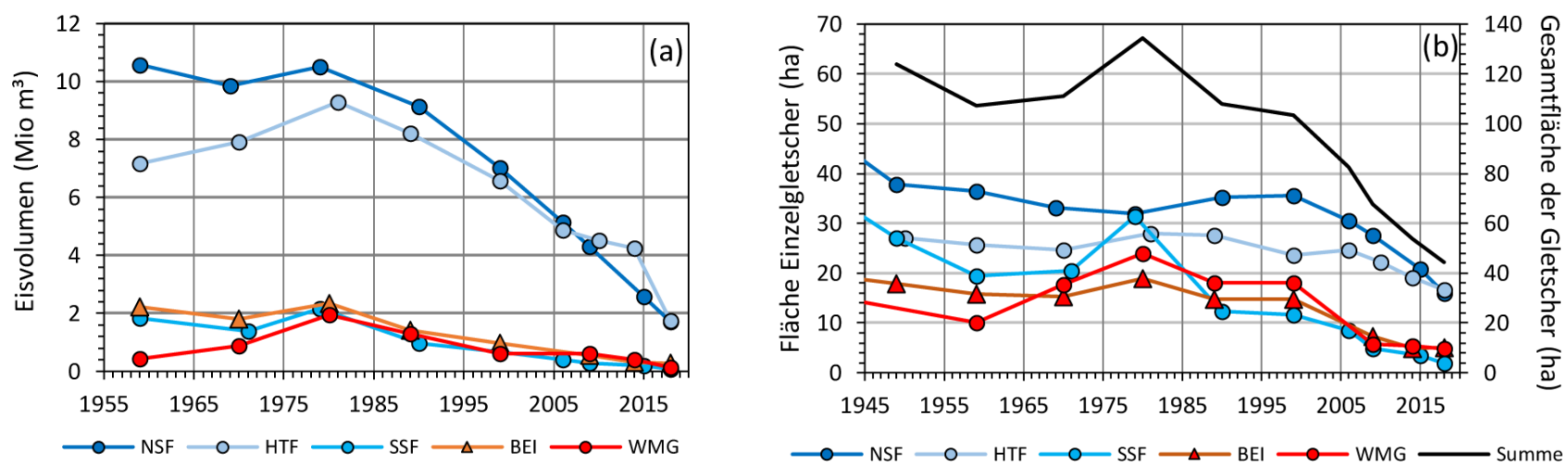

Abb. 3. Entwicklung von Eisvolumen (a) und Gletscherfläche (b) der Bayerischen Gletscher seit der Mitte des 20. Jahrhunderts. Die schwarze Linie zeigt die Gesamtfläche (rechte Achse) seit Mitte des letzten Jahrhunderts.

ferner übriggeblieben. Die maximale Ausdehnung erreichten die bayerischen Gletscher in der ersten Hälfte des 19. Jahrhunderts. Eine topographische Karte wurde 1892 kurz vor der Aufspaltung des Gletschers von Sebastian Finsterwalder erstellt (Abb. 2, Finsterwalder, 1896). Die ersten geometrisch korrekten Karten in den Berchtesgadener Alpen entstanden in den Jahren 1889 für das Blaueis und 1897 für den Watzmanngletscher (Hagg, 2006). Die damals bestimmten Flächen der Gletscher wurden mit 21 ha für den Watzmanngletscher, 20 ha für das Blaueis, 48 ha für den Höllentalferner und 160 ha für den noch zusammenhängenden Schneeferner angegeben.

Ab 1949 begann die systematische und regelmäßige Vermessung aller bayerischen Gletscher, die inzwischen mindestens im 10-Jahresabstand durch die Bayerische Akademie der Wissenschaften in München durchgeführt wird (EscherVetter und Rentsch, 1995; http://www.glaziologie.de, letzter Zugriff: 11. März 2021). Neben der Kartierung der Gletscheroberfläche wurde zwischen 2006 und 2009 auch das Felsbett unter den einzelnen Gletschern als Basis für Eisdickenverteilungen mit Bodenradarmessungen bestimmt. Seit den ersten quantitativen Beobachtungen können die Veränderungen der bayerischen Gletscher über etwa 130 Jahre bestimmt werden.

\section{Die Veränderung der Gletscherflächen}

Die Rekonstruktion der maximalen Gletscherausdehnung in Süddeutschland basiert auf der Kartierung von Endmoränen, z.B. für die späteiszeitliche Ausdehnung bei Burghausen, Memmingen, Kaufbeuren oder in der Nähe des Chiemsees. An den bayerischen Gletschern sind im Gegensatz dazu nur vereinzelt Moränen der Maximalausdehnung zum Ende der kleinen Eiszeit um 1850 zu finden (Hirtlreiter, 1992). Damals bedeckten die Gletscher noch eine Fläche von etwas mehr als $4 \mathrm{~km}^{2}$, wobei etwa $3 \mathrm{~km}^{2}$ auf den Plattachferner entfielen. Nach dessen Aufspaltung wurden bis heute konsequent fünf Gletscher als eigenständige Einheiten identifiziert und

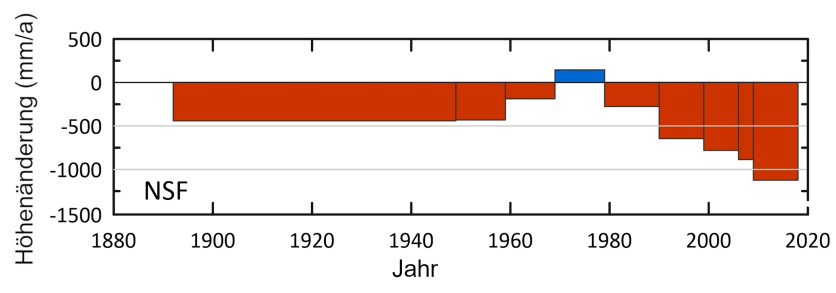

Abb. 4. Die mittleren Höhenänderungen des Nördlichen Schneeferners in Millimetern pro Jahr seit Anfang des 20. Jahrhunderts (rot: Höhenabnahme, blau: Höhenzunahme).

beobachtet. Seit Mitte des 20. Jahrhunderts erfolgen periodische Bestimmungen der Gletscherflächen mit üblichen Vermessungsmethoden. Zusätzlich wurden nach 2000 die Eisdicken aller Gletscher mit Bodenradarmessungen bestimmt. Die Entwicklung der Gletscher seit dem Beginn dieser detaillierten Messungen zeigt einen drastischen Rückgang der Gletscherfläche in den letzten sieben Jahrzehnten von ca. 120 auf 44 ha (Abb. 3). Dieser Rückgang verlief jedoch nicht kontinuierlich. Die meisten Gletscher erreichten um 1960 ein Minimum mit einer Gesamtfläche von etwas mehr als einem Quadratkilometer. Danach steigt diese Fläche bis 1980 wieder auf fast 140 ha an. Erst nach 1980 begann ein allgemeiner Rückgang der Gletscherflächen, der sich bis heute fortsetzt. Der nördliche Schneeferner zeigte vor allem in der letzten Dekade einen starken Rückgang, so dass nun der Höllentalferner der größte bayerische Gletscher ist.

Qualitativ sind die längerfristigen Trends der Flächenveränderungen und insbesondere ihre Summenkurve mit der Abweichung der Lufttemperatur vom langjährigen Mittelwert korreliert. Dies zeigt, dass die Lufttemperatur ein recht guter Indikator für den Klimaeinfluss auf die Gletscher ist (Hagg et al., 2012). Die einzelnen Gletscher weichen dagegen durchaus in ihrem Verhalten ab, was auf lokale Besonderheiten hindeutet. So ist die Flächenzunahme der kleinen Gletscher (SSF, BEI und WMG) bis 1980 sehr viel markanter als bei den größeren Gletschern (NSF und HTF), wo sie erst 


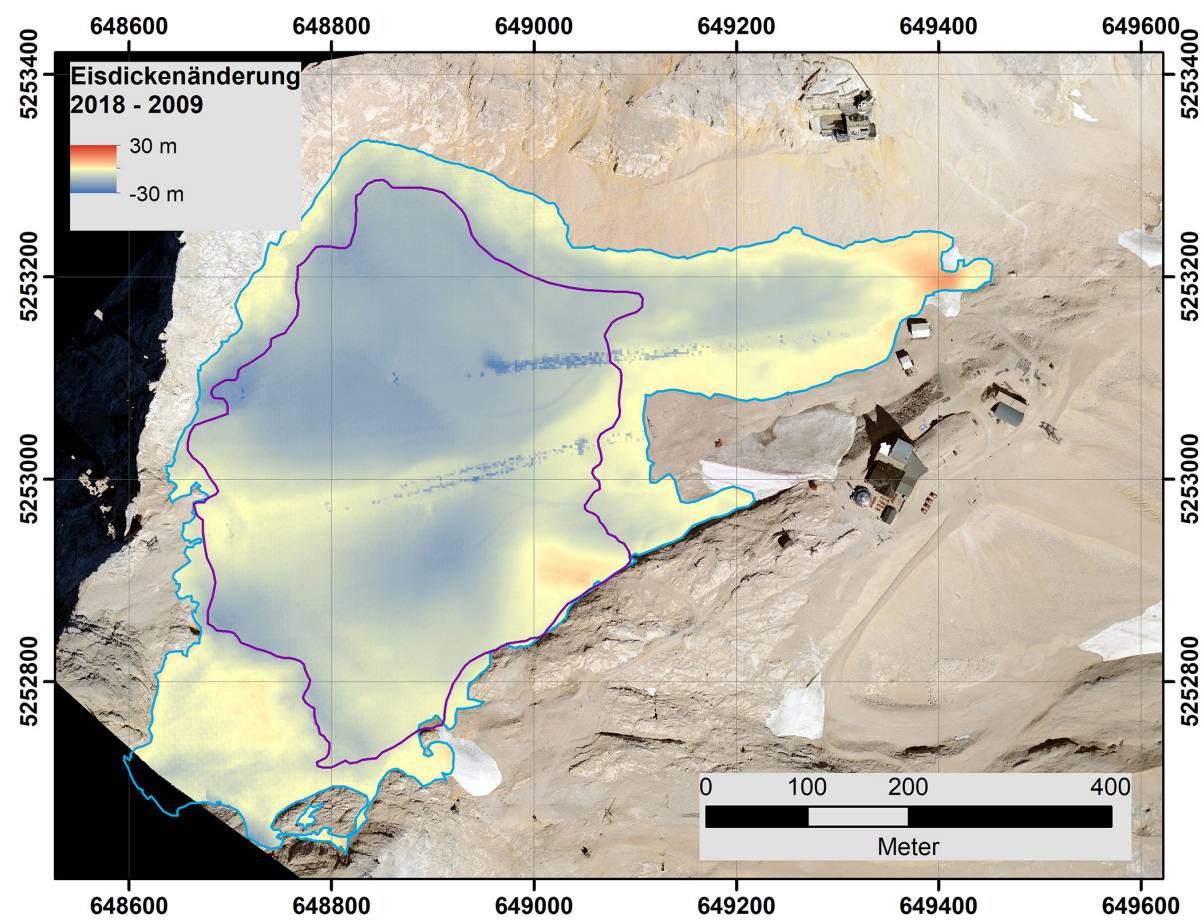

Abb. 5. Eisdickenabnahme des Nördlichen Schneeferners zwischen den Aufnahmezeitpunkten 2009 und 2018. Durch blaue Flächen sind Bereiche mit einer Abnahme der Eisdicke gekennzeichnet. Die Gletschergrenzen sind jeweils für beide Zeitpunkte eingetragen (Hintergrund: Orthofoto 2018).

deutlich verzögert und über einen längeren Zeitraum sichtbar wird. An der Zugspitze zeigt sich in der letzten Dekade eine kontinuierliche Flächenabnahme. Die Gletscher in Berchtesgaden sind dagegen relativ stabil. Im Fall des Blaueises liegt das an der Schuttbedeckung des unteren Teils des Gletschers, welche die Eisschmelze reduziert. Beim Watzmanngletscher lässt seine wannenförmige Einbettung keine großen Flächenänderungen zu. Beide Gletscher erhalten zudem im Gegensatz zu den exponiert gelegenen Gletschern auf dem Zugspitzplatt weniger direkte Sonnenstrahlung.

\section{Entwicklung der Eisdicke}

Die Änderung des Gletschervolumens (Abb. 3a) hat mehr Aussagekraft als die reinen Flächenänderungen (Abb. 3b), da sie in direktem Bezug zur Oberflächenmassenbilanz der Gletscher steht. Die Daten basieren auf den regelmäßigen Oberflächenkartierungen und der Rekonstruktion des Gletscheruntergrunds aus den Bodenradardaten. Allerdings sind diese Messungen auch aufwändiger als die reine Flächenbestimmung, da die Höhenänderungen vor allem an den kleinen Gletschern nur lokal (terrestrisch oder durch Befliegung) bestimmt werden können.

Die durch die Gletscherfläche dividierte Volumenänderung entspricht der mittleren Höhenänderung über dem Gletscher und ist, umgerechnet auf die Dichte von Wasser, damit analog der spezifischen Massenbilanz. In Abb. 4 ist die mitt- lere Höhenänderung pro Jahr für die einzelnen Vermessungsperioden des Nördlichen Schneeferners exemplarisch dargestellt. Seit 1980 verstärkt sich der Massenverlust mit Ausnahme des südlichen Schneeferners und Blaueises an allen Gletschern.

Die flächenhaften Verluste der Eisdicke zeigen nur am Höllentalferner noch ein typisches Muster mit einem ausgeprägten Maximum im unteren Bereich der Gletscherzunge, während an den anderen Gletschern die starken Schmelzraten weitgehend alle Höhenbereiche erfasst haben. Der nördliche Schneeferner (Abb. 5) hat dagegen seine Zunge vollständig verloren und die Eisdickenabnahme entspricht dort der vorher noch vorhandenen Eisdicke. Bereiche mit einem scheinbaren Anwachsen der Eisdicke am Nördlichen Schneeferner sind auf Maßnahmen der Zugspitzbahnen zurückzuführen, welche im südlichen Bereich eine Zugangsrampe zum Gletscher geschaffen und den untersten Zungenbereich zugeschüttet haben (siehe auch Abb. 1b). Die vermeintlichen punktuellen Höhenverluste entlang der Lifttrassen sind dagegen Artefakte. Allerdings deuten die durchgehend geringeren Verluste entlang der südlichen Lifttrasse darufhin, dass hier die Instandhaltung des Skilifts zu einem verminderten Absinken des Gletschers führt. 
$\AA^{N}$
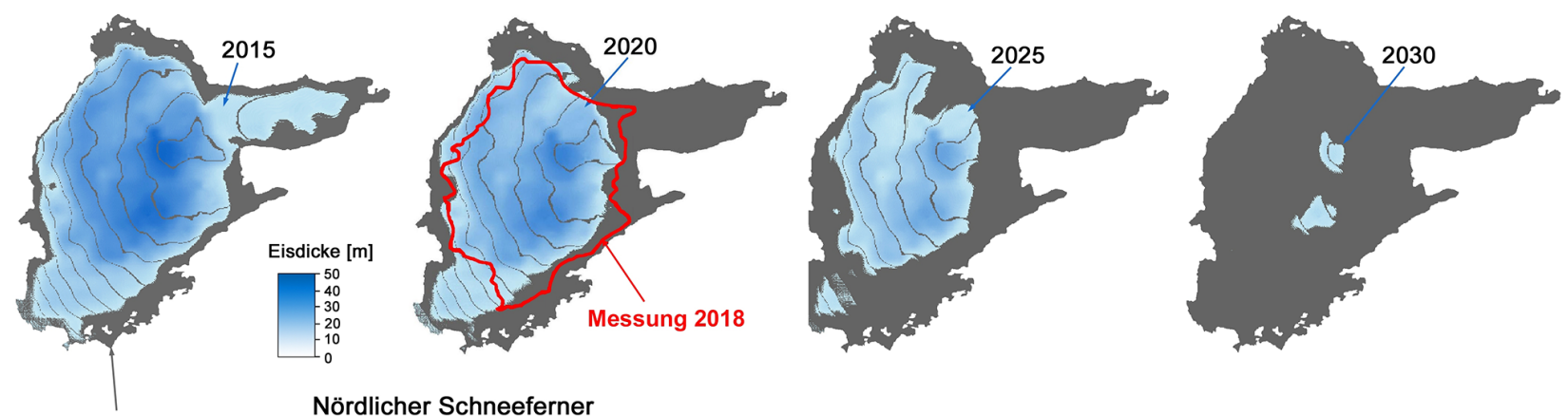

Gletscherfläche 2006

Nördlicher Schneeferner

$\overline{050100 \quad 200300 \quad 400 \mathrm{~m}}$

Abb. 6. Simulation der Flächen- und Eisdickenverteilung des Nördlichen Schneeferners mit dem Gletschermodell SURGES und der Klimasimulation REMO von 2006 bis 2030 (nach Marowsky, 2010). Rot eingetragen sind die Umrisse der Messungen 2018.

\section{Die Zukunft der Bayerischen Gletscher?}

Die Grundlage für alle Schätzungen der zukünftigen Entwicklung der bayerischen Gletscher bildet die räumliche Verteilung der verbliebenen Eisdicken. Unsere Analysen zeigen, dass die maximale Eisdicke 2018 am Nördlichen Schneeferner und am Höllentalferner noch $33 \mathrm{~m}$ betrug, während sie am Blaueis nur noch $17 \mathrm{~m}$ und am Watzmanngletscher und dem Südlichen Schneeferner jeweils noch $10 \mathrm{~m}$ war. Unter Berücksichtigung der erwähnten Eisdickenänderungen zwischen $20 \mathrm{~m}$ in neun Jahren und $30 \mathrm{~m}$ in den 30 Jahren davor und einem Trend zur weiteren Zunahme der Schmelzraten ergibt bereits eine einfache Abschätzung, dass unter den derzeitigen Bedingungen wohl auch die größeren der Bayerischen Gletscher die nächste Dekade kaum überstehen werden. Die Reste der kleineren Gletscher könnten theoretisch gar binnen weniger Jahre verschwunden sein.

Eine komplexere Modellierung existiert für die Zugspitzgletscher (Marowsky, 2010) für den Zeitraum 2006 bis 2030 auf der Basis eines gekoppelten Massenbilanz- und Eisumverteilungsmodells. Als Ausgangsbedingung dienten die Gelände- und Eisdickenmodelle des Jahres 2006. Untersucht wurde die Gletscherentwicklung für drei unterschiedliche Szenarien aus dem GLOWA-Danube Projekt (Mauser und Prasch, 2016): eine Zeitreihe des regionalen Klimamodells REMO des Max-Planck-Instituts für Meteorologie in Hamburg, ein auf der Basis der IPPC Szenarien von 2007 generierter Datensatz und eine einfache Fortschreibung des bisher gemessenen Klimatrends.

Die Ergebnisse mit dem REMO-Datensatz als Randbedingung zeigen unter anderem einen weitgehenden Verlust des nördlichen Schneeferners bis 2030 (Abb. 6).

Die Ergebnisse der Studie von 2010 überdecken 13 Jahre bis heute und weitere zehn Jahre in die Zukunft. Die Eisdickenverteilung, sowie auch die Eisbedeckung, der letzten Vermessung von 2018 für den Nördlichen Schneeferner stimmen sehr gut mit den Modellergebnissen überein. Es ist daher sehr wahrscheinlich, dass zumindest auf dem Zugspitz- platt bis 2030 nur noch wenige Eisreste überleben werden. Entgegen der früheren Entwicklung zeigte auch der Höllentalferner während der letzten Dekade sehr deutliche Massenverluste. Er dürfte zwar den nördlichen Schneeferner um ein paar Jahre überdauern, sein Erscheinungsbild wird sich aber in den nächsten 10 Jahren erheblich wandeln. Damit ist zu erwarten, dass die letzten Eisreserven in den deutschen Alpen in den kommenden Jahrzehnten bis auf wenige Reste verschwinden werden.

Datenverfügbarkeit. Alle Daten sind auf http://www. bayerische-gletscher.de (letzter Zugriff: 11. März 2021) verfügbar.

Autorenmitwirkung. CM entwickelte das Konzept der Studie. Die Analyse der geodätischen Daten wurde von CM und WH durchgeführt. Die Auswertung der Klimadaten und Modellstudien erfolgte durch MW. AW initiierte den Artikel und entwarf den Aufbau. Alle Autoren beteiligten sich am Schreiben des Manuskripts.

Interessenkonflikt. Die Autor*innen erklären, dass kein Interessenkonflikt besteht.

Danksagung. Diese Arbeit wurde unterstützt durch das Projekt „Alpengletscher im Klimawandel“, finanziert durch das Bayerische Staatsministerium für Umwelt und Verbraucherschutz. Unser Dank gilt dem Editor, dessen Kommentare zur Verbesserung und Klarheit des Artikels beitrugen. Für die Unterstützung bei den Feldarbeiten danken wir allen beteiligten Kolleg*innen, insbesondere Astrid Lambrecht.

Begutachtung. This paper was edited by Donovan Dennis. 


\section{Literatur}

Escher-Vetter, H. und Rentsch, H.: The behaviour of the bavarian glaciers: results from surveying and mass balance modelling, Zeitschrift für Gletscherkunde und Glazialgeologie, 31, 181187, 1995

Finsterwalder, R.: Die Gletscher der Bayerischen Alpen. Jahrbuch des Deutschen Alpenvereins, Überbrückungsband der Alpenvereinszeitschrift 1943-1951, Alpiner Verlag Fritz Schmitt, München, 60-66, 1951.

Finsterwalder, S.: Bericht über die Gletscher des Deutschen Reichs 1895, Veröffentlicht von der Commission Internationale des Glaciers in: Les Variations périodiques des glaciers, Premiere Rapport 1895/2, Imprimerie Rey \& Malavallon, Geneva, 129-147, 1896.

Hagg, W.: Digitale Aufbereitung historischer Gletscherkarten in Bayern, Mitteilungen der Geographischen Gesellschaft München, 88, 67-88, 2006.

Hagg, W.: Die Bedeutung kleiner Gletscher am Beispiel der bayerischen Alpen, Geographische Rundschau, 3/2008, 22-29, 2008.
Hagg, W., Mayer, C., Mayr, E., and Heilig, A.: Climate and glacier fluctuations in the Bavarian Alps during the past 120 years, Erdkunde, 66, 121-142, 2012.

Hirtlreiter, G.: Spät- und postglaziale Gletscherschwankungen im Wettersteingebirge und seiner Umgebung, Münchener Geographische Abhandlungen, Reihe B, 15, 176 S., 1992.

Marowsky, K.: Die Validierung des Gletschermodells Surges am Beispiel von Vernagtferner sowie Nördlichem und Südlichem Schneeferner, Diplomarbeit, Kath. Univ. Eichstätt-Ingolstadt, Eichstätt, 147 pp., 2010.

Mauser, W. und Prasch, M.: Regional Assessment of Global Change Impacts - The Project GLOWA-Danube, Springer-Verlag, Berlin, https://doi.org/10.1007/978-3-319-16751-0, 2016.

Mayer, C. und Hagg, W.: Die Entwicklung der bayerischen Gletscher seit der Kleinen Eiszeit, Geographische Rundschau, 5, 1824,2018 . 